Research Paper

\title{
IRF4/MUM1 expression is associated with poor survival outcomes in patients with peripheral T-cell lymphoma
}

\author{
Mi Hwa Heo ${ }^{1,3}$, Ha Young Park², Young Hyeh Ko², Won Seog Kim¹, Seok Jin Kim凶 \\ 1. Division of Hematology-Oncology, Department of Medicine, Samsung Medical Center, Sungkyunkwan University School of Medicine, Seoul, Korea; \\ 2. Department of Pathology, Samsung Medical Center, Sungkyunkwan University School of Medicine, Seoul, Korea. \\ 3. Department of Internal Medicine, Keimyung University Dongsan Medical Center, Daegu, Korea. \\ $\square$ Corresponding author: Seok Jin Kim, MD, PhD, Division of Hematology-Oncology, Department of Medicine, Samsung Medical Center, Sungkyunkwan \\ University School of Medicine, 81 Irwon-ro, Gangnam-gu, Seoul 06351, Korea Tel: +82-2-3410-1766 Fax: +82-2-3410-1754 E-mail: kstwoh@skku.edu \\ (c) Ivyspring International Publisher. This is an open access article distributed under the terms of the Creative Commons Attribution (CC BY-NC) license \\ (https://creativecommons.org/licenses/by-nc/4.0/). See http://ivyspring.com/terms for full terms and conditions.
}

Received: 2016.08.27; Accepted: 2017.01.09; Published: 2017.03.29

\begin{abstract}
Background: Interferon regulatory factor 4 (IRF4)/multiple myeloma oncogene-1 (MUMI) is a member of the interferon regulatory factor family of transcriptional factors. Although IRF4/MUMI expression is associated with aggressiveness of B-cell lymphoma and multiple myeloma, the prognostic value of IRF4/MUMI expression in peripheral T-cell lymphoma (PTCL) is unclear.

Methods: We analyzed a tissue array from 69 patients diagnosed with PTCL. The expression levels of IRF4/MUMI and associated proteins such as MYC and Ikaros were analyzed by immunohistochemistry. Samples were classified by IRF4/MUMI expression into a negative group (less than $5 \%$ of all tumor cells staining positive) or a positive group ( $\geq 5 \%$ of all tumor cells staining positive).

Results: IRF4/MUMI expression was observed in 33\% of all patients (23/69), most frequently in patients with anaplastic large cell lymphoma (ALCL, 78\%, 7/9). Patients with PTCL, not otherwise specified (PTCL-NOS) and angioimmunoblastic T-cell lymphoma (AITL) showed expression rates of $33 \%(9 / 28)$ and $50 \%(4 / 8)$, respectively, whereas only 3 patients with extranodal NK/T-cell lymphoma $(12 \%, 3 / 24)$ showed positive staining. The percentage of IRF4-positive tumor cells was significantly associated with the percentage of MYC-positive tumor cells ( $R$ : $0.410, P=0.013$ ). Comparison of survival outcomes revealed that the IRF4/MUMI-positive group exhibited worse survival than the IRF4/MUMI-negative group; moreover, IRF4/MUMI-positive patients with a high level of MYC expression had the worst survival of all patients with nodal PTCL (PTCL-NOS, AITL, and $\mathrm{ALCL} ; \mathrm{n}=45)(P<0.05)$.

Conclusions: IRF4/MUMI expression was associated with poor survival outcomes in PTCL, implying that this gene is a potential therapeutic target.
\end{abstract}

Key words: IRF4, MUM1, PTCL

\section{Introduction}

Peripheral T-cell lymphomas (PTCLs) are aggressive non-Hodgkin lymphomas (NHLs) characterized by frequent relapse and poor survival outcomes [1]. Most patients with PTCL are still treated mainly with anthracycline-based chemotherapies such as CHOP (cyclophosphamide, doxorubicin, vincristine, and prednisone); however, their treatment outcomes are unsatisfactory compared to those of B-cell lymphomas [2, 3]. Thus, much attention has focused on identifying new therapeutic targets for the development of novel drugs, with the aim of improving PTCL treatment outcomes. Interferon regulatory factor 4 (IRF4), also known as multiple myeloma oncogene-1 (MUM1), has been suggested as a potential therapeutic target in PTCL due to its expression in T-cell lymphoma [4, 5]. IRF4 has been shown to have a crucial role in lymphoid malignancy [6] and is a well-known biomarker of 
activated B-cell like diffuse large B-cell lymphoma [7]. In multiple myeloma, upregulation of IRF4 along with its target gene $M Y C$ is known to contribute to cell proliferation and aggressiveness [8]. Moreover, IRF4 and MYC are targets of lenalidomide because it downregulates IRF4 and MYC via cereblon-dependent destruction of the upstream factor Ikaros [9, 10]. Likewise, lenalidomide has demonstrated clinical activity in relapsed/refractory disease as well as in untreated patients with PTCL [11-13]. Since IRF4 could be a target of other immunomodulatory drugs such as pomalidomide and CC-122, accurately assessing IRF4 expression is potentially important for the future use of novel immunomodulatory drugs in PTCL patients and predicting their treatment outcomes. However, few studies have investigated the clinical and prognostic values of IRF4 or those of its associated proteins (e.g., Ikaros and MYC) in PTCL. Therefore, we analyzed the expression of these proteins and their association with survival outcomes of patients with PTCL.

\section{Patients and methods}

\section{Patients}

We analyzed the protein expression of IRF4, MYC, and Ikaros using a tissue array of samples from patients with PTCL. The tissue array was created using samples from 69 patients who were diagnosed with mature T-cell lymphoma at Samsung Medical Center between January 1994 and December 2004. All tissue samples were reviewed by immunohistochemistry and re-diagnosed according to the 2008 World Health Organization classification. Clinical information data were obtained retrospectively from electronic medical records. The clinical variables assessed were sex, age at diagnosis, PTCL subtype, serum lactate dehydrogenase (LDH) level, extent of extranodal involvement, bone marrow involvement, Ann Arbor stage, International Prognostic Index (IPI) score, and presence of B symptoms. Information collected about induction treatment was type of chemotherapy regimen and response. Final disease relapse and survival status data were recorded at the time of analysis in December 2015.

\section{Immunohistochemistry}

Immunohistochemistry analysis was performed to evaluate the expression of IRF4, MYC, and Ikaros family zinc finger proteins 1 and 3 (IKZF1, IKZF3). Representative 1-mm cores of each case, in duplicate, were taken from tissue blocks after review of slides. Immunohistochemical stains of $4 \mu \mathrm{m}$ paraffin sections of the tissue microarray blocks were performed using a Bond Max automated immunostainer (Leica
Biosystem, Melbourne, Austrailia) using antibodies for IRF4(MUM1p, 1/500; Dako), c-MYC (Y69, cat:ab32072, 1/100; Abcam, Burlingame CA USA,) and IKZF1(LS-C331729-50, 1:1000; LifeSpan BioSciences), IKZF3(LS-C339163, 1:1000; LifeSpan BioSciences). All stained specimens were independently reviewed by a single pathologist (K.Y.H.) without prior knowledge of clinical information. The numbers of positive cells were counted in duplicated cores and positive score was determined in $5 \%$ increments as the percentage of positive tumor cells. As the optimal cutoff value for IRF4 positivity was not defined, we designated positive expression as $\geq 5 \%$ of positive tumor cell, and the staining of less than $5 \%$ of all tumor cells as negative expression. The cutoff for positive expression of MYC protein in lymphoma was variable from $10 \%$ to $40 \%$ among previous studies, and the optimal cutoff was determined according to its statistical association with survival outcomes [14, 15]. Thus, we determined the cutoff for MYC protein expression considering its relation with survival, and defined low expression of MYC as staining of less than $15 \%$ of all tumor cells and high expression as staining of $\geq 15 \%$ of all tumor cells.

\section{Statistics}

Associations of categorical variables were analyzed by the Chi-square test. For continuous variables, the bivariate correlation test with Spearman's rho was performed. For univariate analysis of survival outcomes, the Kaplan-Meier method was used; data were compared using the log-rank test. Overall survival (OS) was measured from the date of diagnosis to the date of death from any cause and was censored at the date of the last follow-up visit. Time to progression (TTP) was assessed from responders to induction chemotherapy and was calculated from the date of diagnosis to the date of disease progression, death from any cause, or the last follow-up. Variables with a $\mathrm{p}$ value $<0.05$ were entered in the analysis, and 95\% confidence intervals (CIs) were calculated. All statistical analyses were performed using PASW Statistics, version 23.0 (SPSS Inc., Chicago, IL, USA).

\section{Results}

\section{Patient characteristics}

The clinical characteristics of the patients are summarized in Table 1 . The median patient age was 54 years (range, 18-86 years), and 44 (63\%) patients were male. More than $60 \%$ of patients had stage III/IV disease, and bone marrow involvement was found in 16 patients $(23 \%)$. The subtype distribution was as follows: peripheral T-cell lymphoma, not otherwise 
specified (PTCL-NOS, $\mathrm{n}=28$ ); extranodal NK/T-cell lymphoma (ENKTL, $\mathrm{n}=24$ ); angioimmunoblastic T-cell lymphoma (AITL, $\mathrm{n}=8$ ); and anaplastic large cell lymphoma (ALCL, $n=9$ ), including two patients with ALK+ALCL. The majority of patients $(78 \%$, 54/69) received CHOP or a CHOP-like regimen as an induction treatment, whereas six patients could not receive induction treatment because they died due to disease progression or combined infections. The overall response rate to induction chemotherapy was $49 \%$, including complete response (24/63) and partial response (7/63). Responses were classified based on the International Working Group response criteria [16]. A total of 25 patients were refractory to induction chemotherapy, including patients with stable disease (2/63) and progressive disease (23/63). Two patients with ALK+ALCL received only one cycle of CHOP treatment: one patient died within two months due to infection and the other patient showed disease progression. Responses were not evaluated in seven patients due to the occurrence of complications such as infection and bleeding. At the time of analysis, the median OS was 12.8 months (95\% CI: 5.8 - 19.8 months).

Table 1. Patient characteristics at diagnosis

\begin{tabular}{ll}
\hline Characteristic & Number (\%) \\
\hline Sex & \\
Male & $44(63)$ \\
Female & $25(37)$ \\
Age & \\
$\leq 60$ years & $43(62)$ \\
> 60 years & $26(38)$ \\
Subtype & \\
PTCL, NOS & $28(40)$ \\
ENKTL & $24(35)$ \\
AITL & $8(12)$ \\
ALCL ALK+/ALK- & $2 / 7(13)$ \\
Stage & \\
I/II & $11 / 14(36)$ \\
III/IV & $13 / 31(64)$ \\
Serum LDH & \\
Normal & $29(42)$ \\
Increased & $40(58)$ \\
Number of involved extranodal lesion & \\
0 & $7(10)$ \\
1 & $36(52)$ \\
$\geq 2$ & $26(38)$ \\
IPI risk & \\
Low & $25(36)$ \\
Low-Intermediate & $12(17)$ \\
High-Intermediate & $21(30)$ \\
High & $11(16)$ \\
Bone marrow involvement & \\
Absent & $16(23)$ \\
Present & \\
B symptoms & \\
Absent & \\
Present & \\
\hline PTCL-NOS, peripheral T-cell lymphoma, not otherwise specified; ENKTL, \\
extranodal NK/T-cell lymphoma; AITL, angioimmunoblastic T-cell lymphoma; \\
ALCL, anaplastic large cell lymphoma; ALK, anaplastic lymphoma kinase; LDH, \\
lactate dehydrogenase; IPI, international prognostic index; BMarrow \\
\end{tabular}

Table 2. Comparison of characteristics between IRF4-negative and IRF4-positive patients with PTCL-NOS, ALCL, or AITL

\begin{tabular}{|c|c|c|c|c|}
\hline \multirow[t]{2}{*}{ Characteristic } & \multirow{2}{*}{$\begin{array}{l}\text { Total } \\
\text { N (\%) }\end{array}$} & \multicolumn{2}{|l|}{ IRF4 } & \multirow[t]{2}{*}{ P value } \\
\hline & & Negative (\%) & Positive (\%) & \\
\hline Sex & & & & 0.759 \\
\hline Male & $28(62)$ & $14(67)$ & $14(58)$ & \\
\hline Female & $17(38)$ & $7(33)$ & $10(42)$ & \\
\hline Age & & & & 0.767 \\
\hline$\leq 60$ years & $27(60)$ & $12(57)$ & $15(62)$ & \\
\hline$>60$ years & $18(40)$ & $9(43)$ & $9(38)$ & \\
\hline Subtype & & & & 0.065 \\
\hline PTCL-NOS & $28(62)$ & $19(81)$ & $9(46)$ & \\
\hline AITL & $8(18)$ & $4(14)$ & $4(21)$ & \\
\hline ALCL & $9(20)$ & $2(5)$ & $7(33)$ & \\
\hline Stage & & & & 0.780 \\
\hline $\mathrm{I} / \mathrm{II}$ & $5 / 8(29)$ & $2 / 3(24)$ & $3 / 5(33)$ & \\
\hline III/IV & $\begin{array}{l}10 / 22 \\
(71)\end{array}$ & $6 / 10(76)$ & $4 / 12(67)$ & \\
\hline Serum LDH & & & & 0.769 \\
\hline Normal & $20(44)$ & $10(48)$ & $10(42)$ & \\
\hline Increased & $25(56)$ & $11(52)$ & $14(58)$ & \\
\hline $\begin{array}{l}\text { Number of involved } \\
\text { extranodes }\end{array}$ & & & & 0.550 \\
\hline 0 & $7(16)$ & $2(9)$ & $3(21)$ & \\
\hline 1 & $21(47)$ & $10(48)$ & $13(46)$ & \\
\hline$\geq 2$ & $17(38)$ & $9(43)$ & $7(33)$ & \\
\hline IPI risk & & & & 0.679 \\
\hline Low & $14(31)$ & $5(24)$ & $9(38)$ & \\
\hline Low-Intermediate & $8(18)$ & $5(24)$ & $3(12)$ & \\
\hline High-Intermediate & $15(33)$ & $7(33)$ & $8(33)$ & \\
\hline High & $8(18)$ & $4(19)$ & $4(17)$ & \\
\hline Bone marrow involvement & & & & 0.329 \\
\hline Absent & $33(73)$ & $17(81)$ & $16(67)$ & \\
\hline Present & $12(27)$ & $4(19)$ & $8(33)$ & \\
\hline B symptoms & & & & 0.373 \\
\hline Absent & $24(53)$ & $13(62)$ & $11(46)$ & \\
\hline Present & $21(47)$ & $8(38)$ & $13(54)$ & \\
\hline \multicolumn{5}{|l|}{ MYC expression } \\
\hline Low & $20(44)$ & $12(57)$ & $8(33)$ & 0.315 \\
\hline High & $16(36)$ & $6(29)$ & $10(42)$ & \\
\hline NE & $9(20)$ & $3(14)$ & $6(25)$ & \\
\hline $\begin{array}{l}\text { PTCL-NOS, peripheral T-cell l } \\
\text { extranodal NK/T-cell lympho } \\
\text { ALCL, anaplastic large cell lyn } \\
\text { international prognostic index }\end{array}$ & $\begin{array}{l}\text { mphoma, } \\
\text { a; AITL, a } \\
\text { phoma; LI } \\
\text { BM, bone }\end{array}$ & $\begin{array}{l}\text { not otherwise sl } \\
\text { angioimmunobla } \\
\text { DH, lactate dehy } \\
\text { marrow; NE, no }\end{array}$ & $\begin{array}{l}\text { ecified; ENKTL } \\
\text { tic T-cell lymp } \\
\text { drogenase; IPI, } \\
\text { evaluated }\end{array}$ & homa; \\
\hline
\end{tabular}

\section{Expression of IRF4, MYC, IKZF1, and IKZF3}

The percentages of IRF4-positive tumor cells ranged from $1 \%$ to $90 \%$ (Figure 1A). A total of 18 patients showed only IRF4 positivity in 1-3\% of all tumor cells, while 24 patients did not show any positively stained cells. Thus, patients were dichotomized into an IRF4-negative or an IRF4-positive group, as follows: positive expression was defined as $\geq 5 \%$ of positive tumor cell staining, whereas staining of less than $5 \%$ of all tumor cells (including no expression) was classified as negative expression. According to this cut-off, 33\% of all patients (23/69) exhibited positive IRF4 expression; these patients most frequently had ALCL $(78 \%, 7 / 9)$, followed by PTCL-NOS $(32 \%, 9 / 28)$ and AITL $(50 \%$, 4/8). Among two patients with ALK+ALCL, one patient was positive for IRF4/MUM1 whereas the 
other patient was negative. However, only three patients with ENKTL showed high expression of IRF4 $(12 \%, 3 / 24)$. MYC expression was also evaluated in 57 patients from whom tissue was available for immunohistochemistry analysis. MYC expression was observed in 53 patients, while four patients showed negative expression (Figure 1B). The percentages of MYC-positive cells varied from $2 \%$ to $90 \%$. Based on our cutoff value of $15 \%$, patients were dichotomized into low expression $(\mathrm{n}=30)$ and high expression $(n=27)$. In contrast, all cases were positive for IKZF1 and IKZF3, and more than $90 \%$ of all tumor cells were positive (Figures 1C, D). As a result, we could not dichotomize patients according to the positive expression of IKZF1 and IKZF3.

\section{IRF4 expression and survival outcomes}

Since positive IRF4 expression was extremely rare in patients with ENKTL, we analyzed the clinical and prognostic value of IRF4 expression only in patients with PTCL-NOS, ALCL, and AITL $(n=45)$. Comparison of clinical characteristics with positive or negative IRF4 expression did not show any significant associations ( $\mathrm{P}>0.05$, Table 2). However, bivariate correlation analysis of IRF4-positive and MYC-positive staining revealed a significant association ( $R$ : $0.410, \mathrm{P}=0.013$ ). The median $\mathrm{OS}$ of the

(A)

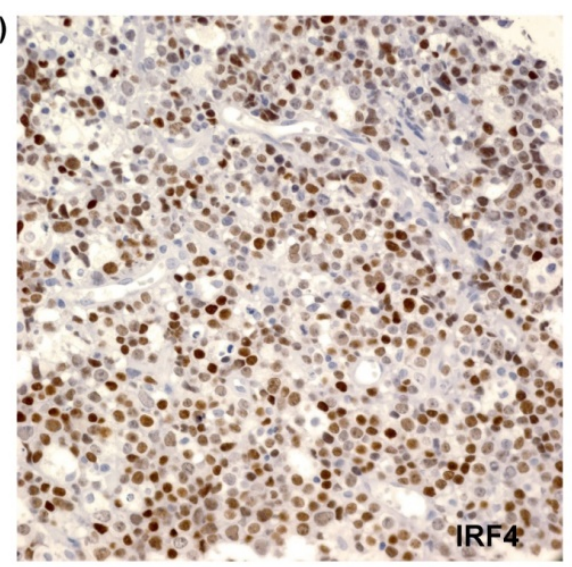

(C)

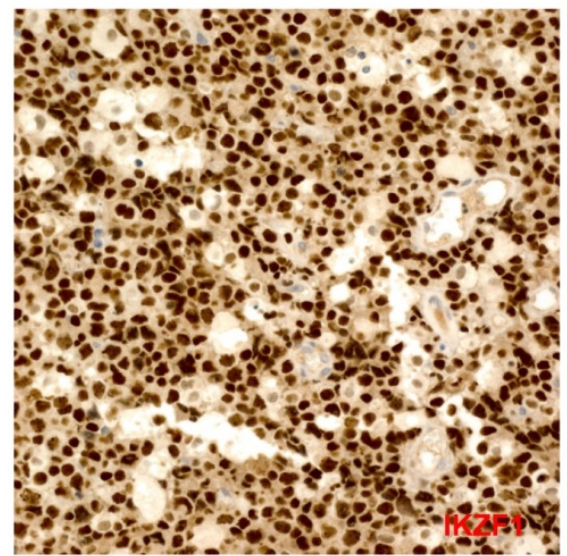

45 patients with nodal PTCL was 15.9 months (95\% CI: 7.4 - 30.4 months). Comparison of TTP and OS according to IRF4 positivity revealed that the TTP of the IRF4-positive group was shorter than that of the IRF4-negative group. Specifically, the median TTP of the IRF4-negative and IRF4-positive groups was 12.6 and 3.1 months, respectively $(P=0.003)$ (Figure $2 \mathrm{~A})$. The median OS of the IRF4-negative group and IRF4-positive group was 21.9 and 5.8 months, respectively; this difference was not statistically significant $(P=0.071) \quad$ (Figure $2 \mathrm{~B})$. The subgroup analysis only with 28 patients with PTCL-NOS also showed the association of IRF4 positivity with survival outcome similar to that of all patients. Thus, the median TTP of the IRF4-negative and IRF4-positive groups was 14.3 and 3.6 months $(P=0.041)$ and the median OS was 24.1 and 8.6 months $(P=0.164)$. Comparison of TTP and OS between the high and low MYC groups did not show any significant differences ( $\mathrm{P}>0.05$, data not shown). However, when the expressions of IRF4 and MYC were combined, the TTP and OS of the IRF4-positive and MYC-high group showed the worst survival, while the IRF4-negative and MYC-low group showed improved survival compared with the other groups (TTP, $P=0.036$; OS, $P=0.026$ ) (Figures 2C, D).

(B)

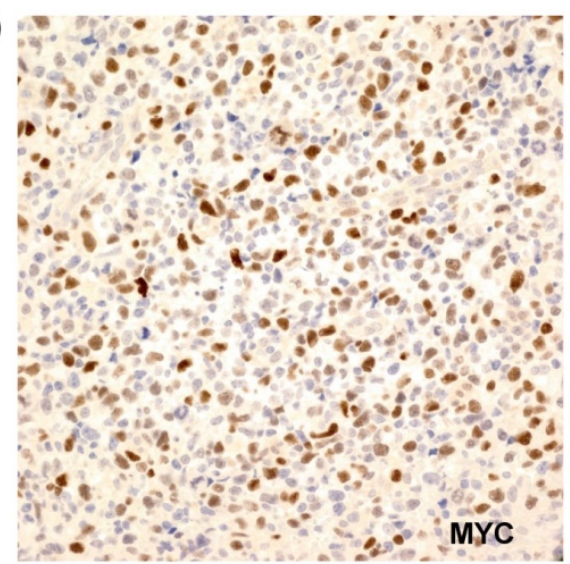

(D)

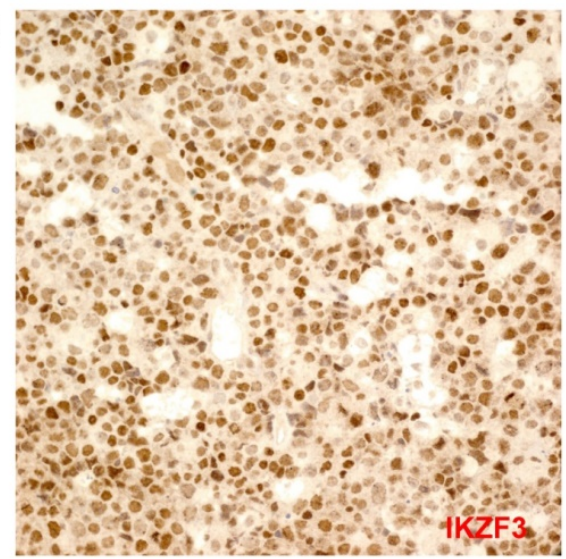

Figure 1. (A-D) Tumor cells are positively stained for IRF4, MYC, IKZFI, and IKZF3 (×400). 
(A)

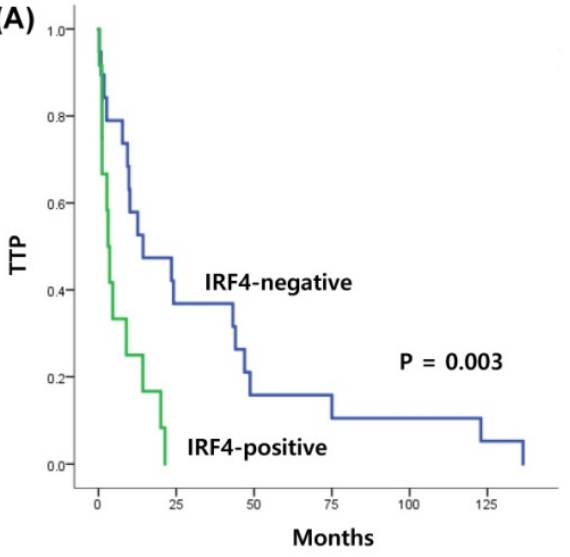

(C)

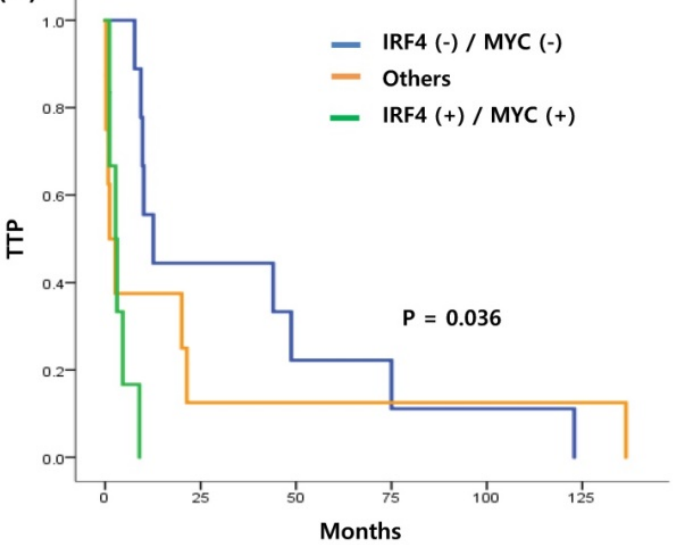

(B)

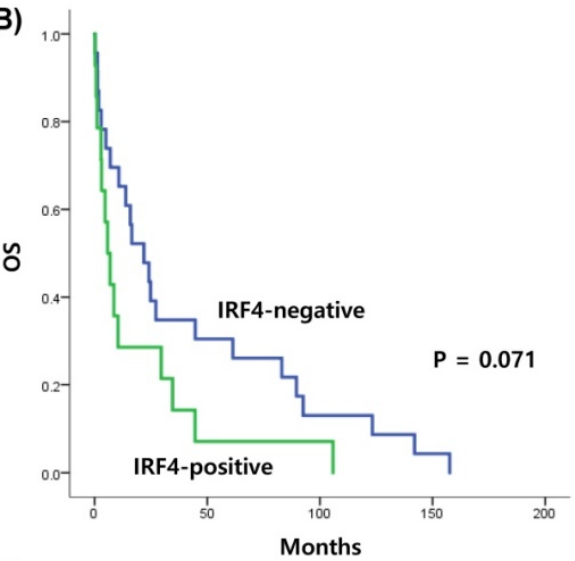

(D)

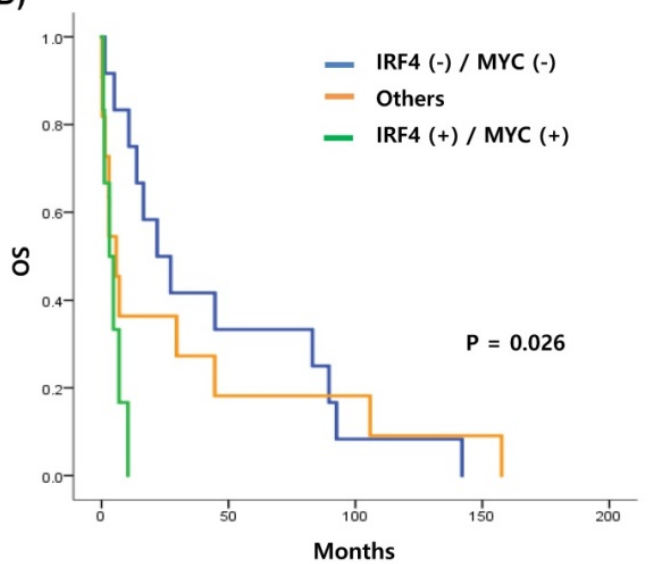

Figure 2. (A, B) Kaplan-Meier curve of TTP and OS in patients with nodal T-cell lymphoma (PTCL-NOS, AITL, and ALCL, $n=45$ ) (C, D) Kaplan-Meier curve of TTP and OS in patients with nodal T-cell lymphoma, stratified by coexpression of IRF4 and MYC (log-rank test $P=0.036$, and $P=0.026$, respectively).

\section{Discussion}

Here we observed IRF4 expression in tumor cells from patients with PTCL. Our results are consistent with those of previous studies that examined IRF4 expression in patients with PTCL $[4,5]$. In this study, we arbitrarily defined the cutoff for positive IRF4 expression as $5 \%$ based on its association with time to progression. Indeed, the association of other cutoff values including $10 \%$ and $30 \%$ with time to progression was not statistically significant $(P>0.05$, data not shown). Our frequency of IRF4 positivity based on the $5 \%$ of cutoff was similar to that of a previous study reporting IRF4 positivity in $42 \%$ $(116 / 277)$ of all patients with PTCL, as assessed by immunohistochemistry [4]. Furthermore, we also observed more frequent IRF4 expression in CD30-positive samples from patients with ALCL because all patients with ALCL were positive for CD30 in our study. This finding is consistent with previous reports showing close association of IRF4 expression with CD30 expression $[4,5]$. However, the positivity for $\mathrm{CD} 30$ was not evaluated in other subtypes of PTCL because CD30 staining was not routinely performed for PTCL patients in clinical practice and our study retrospectively gathered patients who were diagnosed with PTCL. In contrast to patients with nodal PTCL, IRF4 expression was rare in patients with ENKTL $(12 \%, 3 / 24)$ in our study, a finding consistent with a previous study reporting negative expression $(0 / 8)$ [4]. Thus, we analyzed the prognostic value of IRF4 expression in 45 patients with various types of nodal PTCL including PTCL-NOS, ALCL, and AITL (after excluding 23 patients with ENKTL). All these patients with nodal PTCL received $\mathrm{CHOP}$ or a $\mathrm{CHOP}$-like regimen as their first induction treatment; their median overall survival was around 15 months. Clinical characteristics at diagnosis were not significantly associated with IRF4 expression (Table 2). However, comparison of survival outcomes according to IRF4 positivity showed that the IRF4-positive group had worse outcomes than the IRF4-negative group (Figures 2A, B). The percentage of IRF4-positive tumor cells was significantly associated with the percentage of MYC-positive tumor cells (R: 0.410, $\mathrm{P}=0.013$ ); as predicted, patients with IRF4 and MYC expression showed significantly worse TTP and OS than patients without IRF4 and MYC expression (Figures 2C, D). 
Recurrent translocations involving the 6p25 IRF4 locus have been suggested as the underlying mechanism driving IRF4 expression in PTCL [4]. However, fewer cases harbored this translocation than the number of total IRF4-positive cases, as assessed by immunohistochemistry. Therefore, nuclear factor $\mathrm{\kappa B}(\mathrm{NF}-\mathrm{kB})$, which is a candidate regulator of IRF4 expression, has been suggested as another mechanism driving IRF4 expression in PTCL [17]. In PTCL tumor cells, the NF-kB subunits p52 and RelB have been shown to be transcriptional activators of IRF4; moreover, CD30 activation was shown to promote p52 and RelB activity and subsequent IRF4 expression [17]. This positive feedback signal including IRF4, CD30, and NF- $\mathrm{kB}$ is consistent with the higher frequency of IRF4 expression in CD30-positive PTCL subtypes, such as ALCL. Two of the Ikaros proteins, IKZF1 and IKZF3, have been suggested as upstream proteins leading to IRF4 expression in NHL and multiple myeloma [18]. However, all cases in our study showed abundant expression of IKZF1 and IKZF3 (Figures 1C, D). Since $33 \%$ of all patients $(23 / 69)$ were IRF4-positive by immunohistochemistry, our results suggest that IKZF1 and IKZF3 expression are not sufficient to drive IRF4 expression. Interestingly, constitutive expression of IRF4 driving MYC expression was shown to promote proliferation of PTCL cell lines [17]. Moreover, high expression of IRF4 and MYC in ALCL and their essential roles in survival of ALCL cells have been reported [19]. A close association of IRF4 with its direct transcriptional target MYC has mainly been reported in B-cell lymphoid malignancies, including multiple myeloma. Specifically, IRF4 binds directly to the MYC promoter region, thereby activating MYC expression in multiple myeloma cells; this activation leads to cell proliferation $[8,20]$. Signaling cascades such as IRF4, MYC, and NF-kB are molecular targets of immunomodulatory drugs such as lenalidomide [21]. Lenalidomide is effective in the treatment of multiple myeloma, in which IRF4 is expressed, and occasional clinical responses have been reported in patients with PTCL [12, 13, 22, 23]. Accordingly, lenalidomide has shown efficacy in patients with relapsed or refractory PTCL. One phase II study with 23 evaluable patients showed an overall response rate (ORR) of $30 \%$ (all partial responses); the median OS was 7.9 months [23]. Thus, IRF4 is a potential therapeutic target that could also be a predictive factor of response to immunomodulatory drugs in PTCL.

Our study does have some limitations. First, relatively few patients were included, although we note that PTCL is an infrequent disorder. Thus, further studies with larger study populations should be performed to confirm the prognostic value of IRF4 in PTCL. Second, this was a retrospective study reviewing only patients who had tissue array samples available for immunohistochemistry. Thus, only 69 patients were selected from all the patients who were diagnosed and treated between 1994 and 2004. This limitation means that our study may have been vulnerable to selection bias. Lastly, although patients were uniformly treated with anthracycline-based induction chemotherapy at a single institute, the survival outcomes of patients were relatively poor compared to previously reported survival outcomes of PTCL [24, 25]. These poor outcomes could be related to the quality of supportive care, which may have differed from that associated with their current practice. However, despite these limitations, our study indicates that IRF4 expression is significantly associated with survival outcome. Thus, our findings suggest a potential role of IRF4 as a prognostic marker and therapeutic target in PTCL.

\section{Acknowledgements}

This study was supported by a grant from Samsung Medical Center (SMO1150931) and by a grant from the Basic Science Research Program through the National Research Foundation of Korea (NRF), which is funded by the Ministry of Education, Science, and Technology (2014R1A2A1A11049853).

\section{Competing Interests}

The authors have declared that no competing interest exists.

\section{References}

1. Vose J, Armitage J, Weisenburger D. International peripheral T-cell and natural killer/T-cell lymphoma study: pathology findings and clinical outcomes. J Clin Oncol. 2008; 26: 4124-30.

2. Briski R, Feldman AL, Bailey NG, Lim MS, Ristow K, Habermann TM, et al. The role of front-line anthracycline-containing chemotherapy regimens in peripheral T-cell lymphomas. Blood cancer journal. 2014; 4: e214.

3. Ellin F, Landstrom J, Jerkeman M, Relander T. Real-world data on prognostic factors and treatment in peripheral T-cell lymphomas: a study from the Swedish Lymphoma Registry. Blood. 2014; 124: 1570-7.

4. Feldman AL, Law M, Remstein ED, Macon WR, Erickson LA, Grogg KL, et al. Recurrent translocations involving the IRF4 oncogene locus in peripheral T-cell lymphomas. Leukemia. 2009; 23: 574-80.

5. Bisig B, de Reynies A, Bonnet C, Sujobert P, Rickman DS, Marafioti T, et al CD30-positive peripheral T-cell lymphomas share molecular and phenotypic features. Haematologica. 2013; 98: 1250-8.

6. Tsuboi K, Iida S, Inagaki $\mathrm{H}$, Kato $\mathrm{M}$, Hayami $\mathrm{Y}$, Hanamura I, et al. MUM1/IRF4 expression as a frequent event in mature lymphoid malignancies. Leukemia. 2000; 14: 449-56.

7. Hans CP, Weisenburger DD, Greiner TC, Gascoyne RD, Delabie J, Ott G, et al. Confirmation of the molecular classification of diffuse large B-cell lymphoma by immunohistochemistry using a tissue microarray. Blood. 2004; 103: 275-82.

8. Shaffer AL, Emre NC, Lamy L, Ngo VN, Wright G, Xiao W, et al. IRF4 addiction in multiple myeloma. Nature. 2008; 454: 226-31.

9. Lopez-Girona A, Heintel D, Zhang LH, Mendy D, Gaidarova S, Brady H, et al. Lenalidomide downregulates the cell survival factor, interferon regulatory factor-4, providing a potential mechanistic link for predicting response. British journal of haematology. 2011; 154: 325-36.

10. Lu G, Middleton RE, Sun H, Naniong M, Ott CJ, Mitsiades CS, et al. The myeloma drug lenalidomide promotes the cereblon-dependent destruction of Ikaros proteins. Science. 2014; 343: 305-9. 
11. Zinzani PL, Pellegrini C, Broccoli A, Stefoni V, Gandolfi L, Quirini F, et al. Lenalidomide monotherapy for relapsed/refractory peripheral T-cell lymphoma not otherwise specified. Leukemia \& lymphoma. 2011; 52: 1585-8.

12. Morschhauser F, Fitoussi O, Haioun C, Thieblemont C, Quach H, Delarue R, et al. A phase 2, multicentre, single-arm, open-label study to evaluate the safety and efficacy of single-agent lenalidomide (Revlimid) in subjects with relapsed or refractory peripheral T-cell non-Hodgkin lymphoma: the EXPECT trial. European journal of cancer. 2013; 49: 2869-76

13. Toumishey E, Prasad A, Dueck G, Chua N, Finch D, Johnston J, et al. Final report of a phase 2 clinical trial of lenalidomide monotherapy for patients with T-cell lymphoma. Cancer. 2015; 121: 716-23.

14. Valera A, Lopez-Guillermo A, Cardesa-Salzmann T, Climent F, Gonzalez-Barca E, Mercadal S, et al. MYC protein expression and genetic alterations have prognostic impact in patients with diffuse large B-cell lymphoma treated with immunochemotherapy. Haematologica. 2013; 98: 1554-62.

15. Johnson NA, Slack GW, Savage KJ, Connors JM, Ben-Neriah S, Rogic S, et al. Concurrent expression of MYC and BCL2 in diffuse large B-cell lymphoma treated with rituximab plus cyclophosphamide, doxorubicin, vincristine, and prednisone. Journal of clinical oncology : official journal of the American Society of Clinical Oncology. 2012; 30: 3452-9.

16. Cheson BD, Horning SJ, Coiffier B, Shipp MA, Fisher RI, Connors JM, et al. Report of an international workshop to standardize response criteria for non-Hodgkin's lymphomas. NCI Sponsored International Working Group. Journal of clinical oncology : official journal of the American Society of Clinical Oncology. 1999; 17: 1244

17. Boddicker RL, Kip NS, Xing X, Zeng Y, Yang ZZ, Lee JH, et al. The oncogenic transcription factor IRF4 is regulated by a novel CD30/NF-kappaB positive feedback loop in peripheral T-cell lymphoma. Blood. 2015; 125: 3118-27.

18. Kritharis A, Coyle M, Sharma J, Evens AM. Lenalidomide in non-Hodgkin lymphoma: biological perspectives and therapeutic opportunities. Blood. 2015; 125: 2471-6.

19. Weilemann A, Grau M, Erdmann T, Merkel O, Sobhiafshar U, Anagnostopoulos I, et al. Essential role of IRF4 and MYC signaling for survival of anaplastic large cell lymphoma. Blood. 2015; 125: 124-32.

20. Shaffer AL, Emre NC, Romesser PB, Staudt LM. IRF4: Immunity. Malignancy! Therapy? Clinical cancer research : an official journal of the American Association for Cancer Research. 2009; 15: 2954-61.

21. Zhu YX, Kortuem KM, Stewart AK. Molecular mechanism of action of immune-modulatory drugs thalidomide, lenalidomide and pomalidomide in multiple myeloma. Leukemia \& lymphoma. 2013; 54: 683-7.

22. Coiffier B, Federico M, Caballero D, Dearden C, Morschhauser F, Jager U, et al. Therapeutic options in relapsed or refractory peripheral T-cell lymphoma. Cancer treatment reviews. 2014; 40: 1080-8.

23. Dueck G, Chua N, Prasad A, Finch D, Stewart D, White D, et al. Interim report of a phase 2 clinical trial of lenalidomide for T-cell non-Hodgkin lymphoma. Cancer. 2010; 116: 4541-8.

24. Savage KJ. Update: peripheral T-cell lymphomas. Current hematologic malignancy reports. 2011; 6: 222-30.

25. Armitage JO. The aggressive peripheral T-cell lymphomas: 2012 update on diagnosis, risk stratification, and management. American journal of hematology. 2012; 87: 511-9. 